\title{
Formation and stability of cubic ice in water droplets
}

\author{
Benjamin J. Murray and Allan K. Bertram*
}

\author{
Received 22nd September 2005, Accepted 13th October 2005 \\ First published as an Advance Article on the web 2nd November 2005 \\ DOI: $10.1039 / b 513480 c$
}

\begin{abstract}
There is growing evidence that a metastable phase of ice, cubic ice, plays an important role in the Earth's troposphere and stratosphere. Cubic ice may also be important in diverse fields such as cryobiology and planetary sciences. Using X-ray diffraction, we studied the formation of cubic ice in pure water droplets suspended in an oil matrix as a function of droplet size. The results show that droplets of volume median diameter $5.6 \mu \mathrm{m}$ froze dominantly to cubic ice with stacking faults. These results support previous suggestions that cubic ice is the crystalline phase that nucleates when pure water droplets freeze homogeneously at $\sim 235 \mathrm{~K}$. It is also shown that as the size of the water droplets increased from 5.6 to $17.0 \mu \mathrm{m}$, the formation of the stable phase of ice, hexagonal ice, was favoured. This size dependence can be rationalised with heat transfer calculations. We also investigated the stability of cubic ice that forms in water droplets suspended in an oil matrix. We observe cubic ice up to $243 \mathrm{~K}$, much higher in temperature than observed in many previous studies. This result adds to the existing literature that shows bulk ice $\mathrm{I}_{\mathrm{c}}$ can persist up to $\sim 240 \mathrm{~K}$. The transformation of cubic ice to hexagonal ice also showed a complex time and temperature dependence, proceeding rapidly at first and then slowing down and coming to a halt. These combined results help explain why cubic ice forms in some experiments described in the literature and not others.
\end{abstract}

\section{Introduction}

Recently, it was found that cubic ice (ice $\mathrm{I}_{\mathrm{c}}$ ), as opposed to the stable hexagonal phase (ice $\mathrm{I}_{\mathrm{h}}$ ), was the major product when aqueous solution droplets froze homogeneously at temperatures of less than $200 \mathrm{~K}$. ${ }^{1}$ This strongly suggests ice $\mathrm{I}_{\mathrm{c}}$ forms in the Earth's upper troposphere where it may significantly impact the formation of ice clouds and enhance dehydration through a process analogous to the well known BergeronFindeisen process. $^{2}$ This process is driven by the vapour pressure difference between ice $I_{c}$ and ice $I_{h}$, where the metastable phase necessarily has a larger vapour pressure than that of the stable phase. ${ }^{2}$ Ice $I_{c}$ may also be important in other areas such as cryobiology and planetary sciences. ${ }^{3}$

In our recent study on cubic ice we observed that a small amount of ice $I_{c}$ was formed when emulsified pure water droplets (water droplets suspended in an oil matrix) homogeneously froze at $\sim 235 \mathrm{~K}$. ${ }^{1}$ This was unexpected as prior to our work, ice $\mathrm{I}_{\mathrm{c}}$ was only observed in pure water droplets when they were hyperquenched onto a cold substrate below $190 \mathrm{~K} .{ }^{4}$ Ice $I_{c}$ has also been observed when water clusters $(6.6-5.5 \mathrm{~nm})$ froze at $200 \mathrm{~K} .{ }^{5}$ Others have suggested that crystallisation of water droplets begins with nuclei having a cubic structure even at temperatures well above $200 \mathrm{~K}^{5-9}$ Clearly, more work is required to understand the conditions that are required for the formation of ice $I_{c}$.

To explain our previous results and better understand the conditions at which cubic ice forms in pure water droplets we have carried out two sets of experiments: first, we have

Department of Chemistry, University of British Columbia, 2036 Main Mall, Vancouver, British Columbia, Canada V6T $1 Z 1$ investigated the formation of cubic ice in emulsified water droplets as a function of droplet size. Second, we have investigated the stability of ice $\mathrm{I}_{\mathrm{c}}$ that forms in these emulsified droplets. One of the benefits of our experimental configuration is that mass transfer via the vapour phase is blocked since the droplets are suspended in an oil matrix. This allows us to focus on the solid state transformation in contrast to many previous measurements. Our combined studies provide insight into the nucleation and crystallization process, and allow us to speculate on the structure of the crystalline nucleus responsible for homogeneous nucleation in water droplets, an important topic that still is not resolved. Our results, combined with previous results, also allow us to speculate why cubic ice forms in some experiments, which are recorded in the literature, and not in others.

\section{Experimental}

The X-ray diffractometer (Bruker D8 Discover) employed in this study was configured in a standard Bragg-Brentano reflection geometry and was equipped with a $\mathrm{Cu} \mathrm{K} \alpha \mathrm{X}$-ray source and a Bruker SOL-X X-ray detector. In order to improve the signal-to-noise a different source and detector to those described by Murray et al. ${ }^{1}$ were employed in the present study. Other than a factor of two improvements in signal-tonoise, the diffraction patterns from the present and previous studies are directly comparable.

Emulsions of pure water droplets were prepared by mixing pure water (distilled water further purified with a Millipore system) with an oil phase in a proportion of 30-40\% water in oil (by mass). The oil phase consisted of $\sim 10 \mathrm{wt} \%$ of surfactant (lanolin, Aldrich Chemical Company) in 
hydrocarbon oil (paraffin oil, Fisher Scientific, kinematic viscosity at $40{ }^{\circ} \mathrm{C}=34.5 \mathrm{cSt}$ ). This mixture was then agitated for 5-10 min or until the droplets were of the desired size. Droplet size could be varied by adjusting the agitation time and the resulting droplet size distribution was determined by optical microscopy (see the insert in Fig. 2 for an example of a size distribution determined by this method).

The X-ray diffraction experiments were performed with a modified commercial low temperature X-ray diffraction chamber (Anton-Paar, TTK 450). The emulsions were placed in a cell consisting of an aluminium base and covered with a $30 \mu \mathrm{m}$ thick film of Teflon to hold the emulsions in place and also prevent evaporation of the droplets. The temperatures of the cell and emulsion were measured with a thermistor (Pt-100) positioned within the aluminium base. This cell was placed in good thermal contact with a cryostat, which was cooled with a flow of liquid nitrogen and the required temperature set by use of a heater and temperature controller (Anton-Paar, TCU). This system allowed the temperature of the emulsion cell to be set to between 90 and $300 \mathrm{~K}$ (and to higher temperatures if the liquid nitrogen flow was stopped). The uncertainty in the temperature was $\pm 1 \mathrm{~K}$ based on melting point measurements. The temperature of the cell could be ramped up or down at rates up to $30 \mathrm{~K} \mathrm{~min}^{-1}$, where $10 \mathrm{~K} \mathrm{~min}^{-1}$ was the standard cooling rate in the freezing experiments. The cryostat and emulsion cell were positioned inside an airtight chamber which could either be evacuated or purged with a dry flow of $\mathrm{N}_{2}$ in order to prevent frosting at low temperatures. Windows made of Capton in the walls of this chamber permitted X-ray radiation to pass into and out of the chamber.

Since the droplets were suspended in an oil matrix preferred orientation of the crystals after freezing was not expected. Also, the measured diffraction patterns were expected to be equivalent to a powder X-ray pattern, since there were between $10^{6}$ and $10^{8}$ individual frozen droplets exposed to the $\mathrm{X}$-ray beam in a typical experiment. To test these assumptions, we compared the diffraction pattern of the frozen droplets after annealing, which results in pure hexagonal ice (see Fig. 1a), with patterns of hexagonal ice calculated using the POWDER CELL programme ${ }^{10}$ using crystallographic data for hexagonal ice. ${ }^{11}$ The measured peak intensities were in excellent agreement with the calculations, indicating preferred orientation was not an issue. If there was a preferred orientation, or not enough frozen droplets to approximate a powder, the peak intensities would not match the calculations.

\section{Results and discussion}

\section{The formation of ice $I_{c}$ in pure water droplets as a function of droplet size}

Emulsions of pure water droplets were cooled to $173 \mathrm{~K}$ at a rate of $10 \mathrm{~K} \mathrm{~min}^{-1}$, while monitoring a strong ice reflection (at either $2 \theta \approx 24$ or $40^{\circ}$ ) in order to determine the freezing temperatures of the droplets. Freezing of droplets occurs over a range of temperatures, since nucleation is a stochastic process. ${ }^{12}$ The observed freezing range of pure water droplets (between approximately $237.5 \pm 1$ and $230.4 \pm 1 \mathrm{~K}$ ) is in very good agreement with literature values for homogeneous freez-

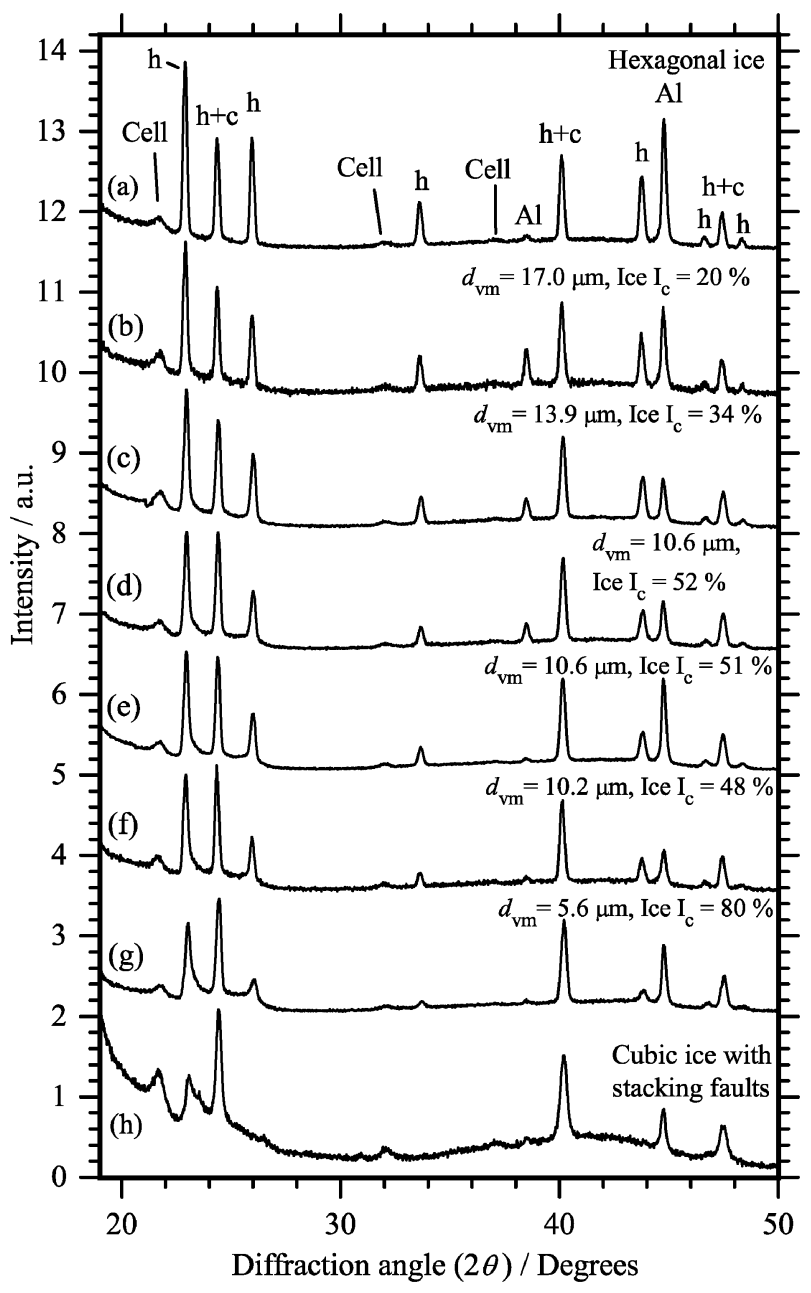

Fig. 1 Diffraction patterns of frozen droplets. Patterns (b) to (g) are the diffraction patterns of frozen pure water droplets, where the size of the droplets was varied. The volume median diameter, $d_{\mathrm{vm}}$, is given for each pattern. The droplets were cooled at a rate of $10 \mathrm{~K} \mathrm{~min}^{-1}$ and froze between $237.5 \pm 1$ and $230.4 \pm 1 \mathrm{~K}$. The pattern shown in (a) is for the same frozen water droplets that appear in (f), but which were annealed at $263 \mathrm{~K}$ for $5 \mathrm{~min}$ to yield pure ice $\mathrm{I}_{\mathrm{h}}$. The diffraction pattern shown in (h) is a diffraction pattern of ice $\mathrm{I}_{\mathrm{c}}$ with stacking faults. This pattern was obtained by freezing $45.2 \mathrm{wt} \%\left(\mathrm{NH}_{4}\right)_{3} \mathrm{H}\left(\mathrm{SO}_{4}\right)_{2}$ solution droplets, which froze between $192.4 \pm 1$ and $184.5 \pm 1 \mathrm{~K}$. The estimated proportion of stacking faulty ice $I_{c}$, determined from the linear combination of the hexagonal patterns (a) and cubic pattern (h), are given for patterns (b) to (g) (see text and Fig. 3 for details of this estimate). All patterns are normalized to the intensity of the peak at $40^{\circ}$ and are shifted on the intensity scale for clarity. Reflections unique to ice $I_{h}$ are labelled " $h$ " and those common to ice $I_{c}$ and ice $I_{h}$ are labelled " $h+c$ ". Bragg peaks from the aluminium base and those from the cell construction materials are labelled $\mathrm{Al}$ and cell, respectively. All diffraction patterns were recorded at $173 \mathrm{~K}$. The baseline in pattern (h) is more pronounced compared with the other patterns because of the smaller water content in the concentrated $\left(\mathrm{NH}_{4}\right)_{3} \mathrm{H}\left(\mathrm{SO}_{4}\right)_{2}$ solution droplets.

ing of micrometer sized droplets, ${ }^{12}$ indicating that the oil and surfactant were not significantly altering the nucleation process and that these droplets froze homogeneously. The observed freezing range varied by less than the uncertainty in the 
measurements as the volume median diameter varied from 5.6-17.0 $\mu \mathrm{m}$. This is consistent with freezing temperatures recorded in the literature. ${ }^{12}$

Once cooled to $173 \mathrm{~K}$ the diffraction patterns of the frozen droplets were measured between $2 \theta=19$ and $50^{\circ}$. Patterns are illustrated in Fig. 1b-g for a number of emulsion samples of varying droplet size. The peaks exclusive to ice $I_{h}$ have been labelled " $h$ " and the peaks common to both ice $I_{c}$ and ice $I_{h}$ have been labelled " $h+c$ ". Also shown for comparison is the diffraction pattern of pure ice $I_{h}$, generated by annealing pure water droplets at $263 \mathrm{~K}$ (Fig. 1a) and a diffraction pattern of ice $\mathrm{I}_{\mathrm{c}}$ (Fig. 1h). Pattern $1 \mathrm{~h}$ was obtained by freezing $45.2 \mathrm{wt} \%$ $\left(\mathrm{NH}_{4}\right)_{3} \mathrm{H}\left(\mathrm{SO}_{4}\right)_{2}$ solution droplets, which froze between $192.4 \pm 1$ and $184.5 \pm 1 \mathrm{~K}$. This diffraction pattern is in very good agreement with the diffraction pattern of ice $\mathrm{I}_{\mathrm{c}}$ reported in the literature. ${ }^{1,3,4,13}$ Note that in the $45.2 \mathrm{wt} \%$ solutions, $\left(\mathrm{NH}_{4}\right)_{3} \mathrm{H}\left(\mathrm{SO}_{4}\right)_{2}$ did not crystallize, and hence in the diffraction pattern only peaks due to ice are observed. The major ice $I_{h}$ reflections at 34 and $44^{\circ}$ are completely absent from this diffraction pattern, indicating the absence of bulk $\mathrm{I}_{\mathrm{h}}$. The peak at $23^{\circ}$, which is usually associated with the (100) reflection of ice $I_{h}$, is present in the diffraction pattern. This feature has been observed in previous studies of ice $I_{c}$ employing X-ray diffraction $^{1,3,4,13}$ and neutron diffraction ${ }^{14-16}$ and has been associated with hexagonal-like stacking faults, believed to be an intrinsic property of ice $\mathrm{I}_{\mathrm{c}} \cdot{ }^{3,14}$ Also, the region between 22 and $27^{\circ}$ is raised above the background. This broad feature is also most likely related to stacking faults. ${ }^{17,18}$

Based on Fig. 1, the diffraction pattern of frozen water droplets with a volume median diameter of $5.6 \mu \mathrm{m}$ (pattern g) has some similarities with the diffraction pattern for ice $I_{c}$ with stacking faults (pattern $h$ ). First, the major ice $I_{h}$ reflections at $\sim 34$ and $44^{\circ}$ are significantly reduced in intensity relative to the peaks common to both ice $\mathrm{I}_{\mathrm{h}}$ and ice $\mathrm{I}_{\mathrm{c}}$. Also, the region between $2 \theta \approx 22.5$ and $26.5^{\circ}$ is significantly raised above the background, similar to ice $\mathrm{I}_{\mathrm{c}}$ with stacking faults.

As the volume median diameter was increased from 5.6 to $17.0 \mu \mathrm{m}$, the peaks exclusive to ice $I_{h}$ increase in intensity relative to the peaks common to both ice $I_{c}$ and ice $I_{h}$ (see patterns $b-g$ in Fig. 1). This indicates that there is a strong size dependence of the ice crystal structure with droplet size, and the amount of ice $I_{c}$ decreases with an increase in droplet size. Overall, as the size increases, the diffraction pattern becomes more like the diffraction pattern of ice $I_{h}$.

The intensity ratios $I_{44} / I_{40}$ and $I_{33} / I_{47}$ (where $I_{44}$ and $I_{33}$ are the intensities of the exclusive hexagonal peaks at $2 \theta \approx 43.5$ and $33.4^{\circ}$, and $\mathrm{I}_{40}$ and $\mathrm{I}_{47}$ are peak intensities common to cubic and hexagonal ice at $2 \theta \approx 40.1$ and $47.1^{\circ}$ ) for the X-ray diffraction patterns illustrated in Fig. 1 have been plotted in Fig. 2 as a function of droplet size. These intensity ratios provide a convenient qualitative measure of the amount of ice $I_{h}$ in the frozen droplets, where $I_{44} / I_{40}=0.79 \pm 0.3$ and $I_{33} / I_{47}=1.31 \pm 0.06$ indicates pure ice $I_{h}$ and a value of zero indicates stacking faulty ice $I_{c}$ (the ratios for pure hexagonal ice are determined from the hexagonal pattern illustrated in Fig. 1a).

The droplet diameter quoted in Fig. 1 and plotted in Fig. 2 is the volume median diameter $\left(d_{\mathrm{vm}}\right)$. The horizontal bars represent the particle diameter range over which $68 \%$ of the volume resides. These bars were calculated from the measured

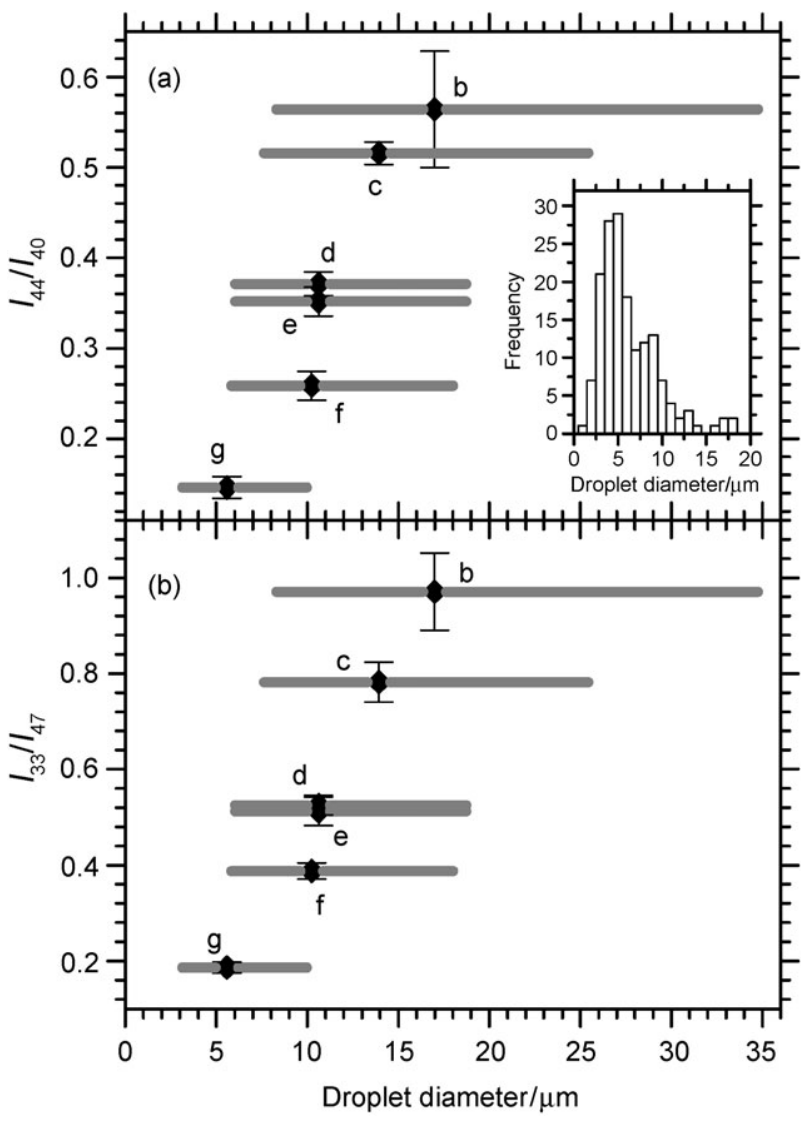

Fig. 2 The intensity ratio $I_{44} / I_{40}$ (panel (a)) and $I_{33} / I_{47}($ panel (b)) as a function of the droplet volume median diameter $\left(d_{\mathrm{vm}}\right)$, which was determined from the measured droplet size distributions. A value of $I_{44} / I_{40}$ or $I_{33} / I_{47}=0$, indicates that no bulk ice $I_{\mathrm{h}}$ formed in the droplets, and therefore that the dominant product was ice $\mathrm{I}_{\mathrm{c}}$, whereas a value of $I_{44} / I_{40}=0.79 \pm 0.03$ or $I_{33} / I_{47}=1.31 \pm 0.06$ indicates pure ice $I_{h}$ (these values were determined from the diffraction pattern of hexagonal ice illustrated in Fig. 1a). The horizontal bars represent the range of droplet sizes in which $68 \%$ of the volume resides. The vertical error bars are derived from the uncertainty associated with measuring the diffraction peak areas. The bracketed letters correspond to the diffraction patterns in Fig. 1. An example of a size distribution is shown as an insert; $d_{\mathrm{vm}}$ for these droplets was $10.6 \mu \mathrm{m}$ with a geometric standard deviation of 1.8 .

geometric standard deviation. ${ }^{19}$ Fig. 2 illustrates the very strong dependence of the phase of ice on droplet size - as the size of the particles increases the amount of ice $I_{c}$ decreases. When the volume median diameter is $5.6 \mu \mathrm{m}$, the droplets freeze close to pure ice $I_{c}$ with stacking faults. Recently, based on classical thermodynamic calculations, it has been suggested that pure water droplets smaller than $30 \mathrm{~nm}$ in diameter would freeze to cubic ice and bigger droplets would freeze to hexagonal ice. ${ }^{20}$ These calculations do not agree with our observations that water droplets with a volume median diameter of $5.6 \mu \mathrm{m}$ freezes dominantly to ice $I_{c}$.

The decrease in the amount of ice $I_{c}$ as the size is increased from $5.6 \mu \mathrm{m}$ can be explained with heat transfer calculations. When a water droplet freezes heat is produced, since crystallization is an exothermic process. If the heat is not dissipated 
to the droplet's environment more rapidly than it is produced during crystallization, the temperature of the droplet will increase during freezing, which can allow ice $I_{c}$ regions in the ice droplet to anneal to ice $\mathrm{I}_{\mathrm{h}}$ (it will be shown later in this paper that the ice $I_{c}$ to ice $I_{h}$ transition occurs more readily at higher temperatures). Based on equations given in Pruppacher and Klett, ${ }^{12}$ and temperatures and thermal properties consistent with our experiments, a droplet of $10 \mu \mathrm{m}$ in diameter will dissipate heat to the oil matrix at a rate similar to the rate of heat production within the supercooled droplet due to crystallization. As a result $10 \mu \mathrm{m}$ particles may not warm up sufficiently to anneal all ice $I_{c}$ to ice $I_{h}$, which is consistent with our experimental results. Smaller droplets will have a greater surface area to volume ratio than larger droplets and will therefore dissipate heat more efficiently. Hence, one would expect the amount that the particle warms up, and therefore the amount of ice $I_{c}$, to depend strongly on droplet size, which is in agreement with our observations. Our combined results are consistent with ice $I_{c}$ nucleating in all of the droplets, and the final amount of ice $I_{c}$ being governed by the amount the temperature of the droplet increases during freezing. From this we infer that ice $I_{c}$, rather than ice $I_{h}$, is likely to be the crystalline phase that nucleates when water droplets freeze homogeneously at $\sim 235 \mathrm{~K}$. In making this statement, we assume that the crystalline phase of the ice nucleus is the same as the crystalline phase observed in the small droplets, which experience no appreciable heating. This is similar to assumptions that have been previously made in the literature. ${ }^{5}$ This also assumes that crystallization begins with the formation of a critical nucleus with a well defined crystal structure, which is still a matter of debate in the literature. ${ }^{21}$

Our inference that homogeneous nucleation of water droplets at $\sim 235 \mathrm{~K}$ begins with ice $\mathrm{I}_{\mathrm{c}}$ is consistent with thermodynamic arguments that indicate the free energy of formation of an octahedral germ of ice $I_{c}$ is lower than the free energy of formation of an ice $\mathrm{I}_{\mathrm{h}}$ germ. ${ }^{8,22}$ Also these results are consis- tent with previous experiments by Huang and Bartell. ${ }^{5}$ These authors froze water clusters $(5.5-6.6 \mathrm{~nm})$ at $200 \mathrm{~K}$ in a supersonic expansion and determined the free energy of the solid-liquid interface when ice nucleated in these clusters. They found that the clusters froze to ice $I_{c}$, using electron diffraction, and they also noticed that the interfacial energies they determined were consistent with those determined from homogeneous freezing experiments of emulsified water droplets. Based on this they concluded that ice $\mathrm{I}_{\mathrm{c}}$ initially nucleates in water droplets at $\sim 235 \mathrm{~K}$ as well as at $200 \mathrm{~K}$. Furthermore, it has been suggested that ice $I_{c}$ is the phase that nucleates based on measurements of the angles between the $c$-axis in snow polycrystals and frozen water droplets. ${ }^{6,7}$ These previous measurements combined with our direct measurements of the phase of ice that forms in water droplets provides convincing evidence that ice $\mathrm{I}_{\mathrm{c}}$ is the crystalline phase that nucleates when pure water droplets freeze homogeneously at $\sim 235 \mathrm{~K}$.

In Fig. 3, we have compared experimental diffraction patterns of frozen water droplets with composite patterns generated by taking a linear combination of the pattern of pure ice $I_{h}$ (Fig. 1a) and ice $I_{c}$ with stacking faults (Fig. 1h). The best fits were determined by minimizing the sum of squares differences between the experimental and composite patterns. The agreement between measured and composite patterns (open circles and solid lines, respectively) is good. The good agreement suggests that pure water droplets may freeze to a combination of ice $I_{h}$ and stacking faulty ice $I_{c}$. The proportions of ice $I_{c}$ with stacking faults and ice $I_{h}$ determined from this analysis have been quoted in Fig. 3. This analysis has also been applied to the other diffraction patterns in Fig. 1, and the proportions of stacking faulty ice $\mathrm{I}_{\mathrm{c}}$ from this analysis are also quoted there. When estimating the proportion of stacking faulty ice $I_{c}$ and ice $I_{h}$ it was assumed that the X-ray quantitation constants (integrated intensity per unit mass of ice) for the common peaks are the same for hexagonal ice and cubic ice

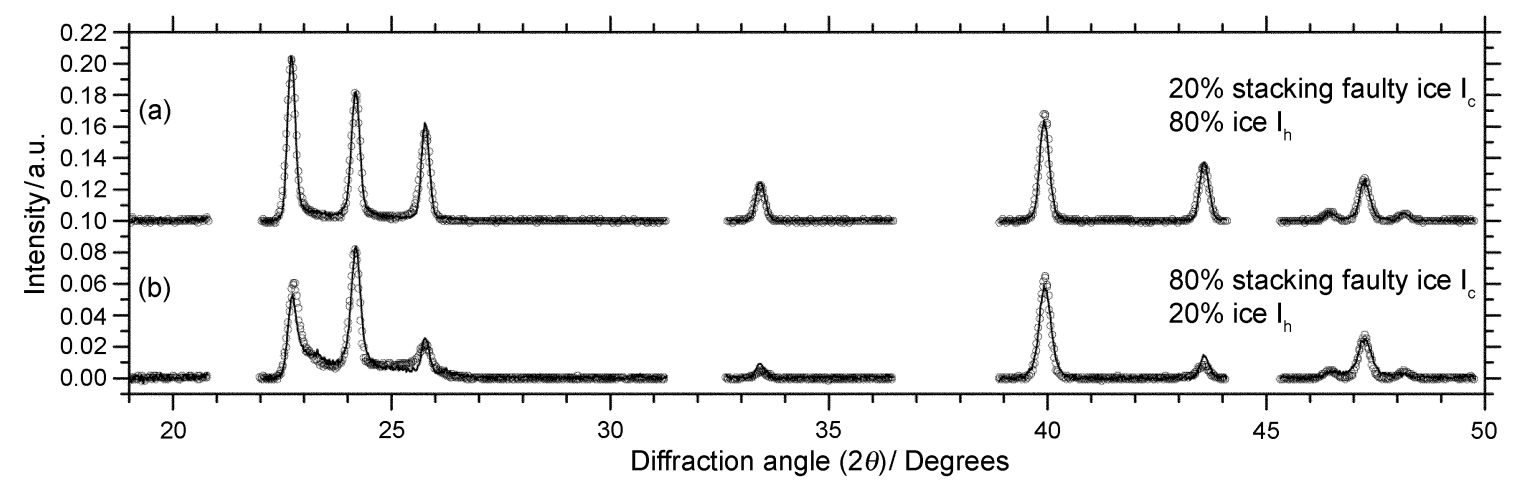

Fig. 3 Comparison of composite (solid lines) and measured X-ray diffraction patterns (points). The composite patterns result from a linear combination of two separate patterns: the first is a pattern of $45.2 \mathrm{wt} \%\left(\mathrm{NH}_{4}\right)_{3} \mathrm{H}\left(\mathrm{SO}_{4}\right)_{2}$ solution droplets which froze at around $188 \mathrm{~K}$ to stacking faulty ice $I_{c}$ (illustrated in Fig. 1h); the second diffraction pattern (illustrated in Fig. 1a) is that of pure water which froze around $235 \mathrm{~K}$ and was subsequently annealed at $263 \mathrm{~K}$ to form ice $\mathrm{I}_{\mathrm{h}}$ with no detectable stacking faults or regions of ice $\mathrm{I}_{\mathrm{c}}$. The pure ice $\mathrm{I}_{\mathrm{h}}$ pattern and the stacking faulty ice $I_{c}$ pattern were scaled and added together to give the composite patterns. The scaling factors were determined by minimizing the sum of squares differences between the composite and experimental patterns and the resulting proportions of stacking faulty cubic ice and hexagonal ice are indicated in the figure (assumptions and uncertainties associated with these values are discussed in the text). The measured patterns are (a) droplets of $d_{\mathrm{vm}}=13.9 \mu \mathrm{m}$ (Fig. 1c) and (b) droplets of $d_{\mathrm{vm}}=5.6 \mu \mathrm{m}$ (Fig. 1g). All patterns were background subtracted. The regions of the diffraction patterns influenced by diffraction from the cell construction materials were not included when calculating the sum of squares differences and have been removed for clarity. 
with stacking faults. Measurements in which we monitor the intensity of the common peaks as cubic ice with stacking faults is annealed to hexagonal ice show that the X-ray quantitation constants are the same to within 5\%. Note that the quantitation constants of the common peaks for pure cubic ice (samples free of stacking faults) will be different from the quantitation constants for hexagonal ice, based on calculations of the diffraction patterns using the POWDER CELL programme. ${ }^{10}$ Evidently stacking faults appear to influence the quantitation constants of cubic ice. Assuming a $10 \%$ uncertainty in quantitation constants, the proportions of stacking faulty ice $\mathrm{I}_{\mathrm{c}}$ and ice $\mathrm{I}_{\mathrm{h}}$ only change by at most $5 \%$.

In the future we will carry out a full modelling study of the diffraction patterns to quantitatively evaluate the proportions of ice $I_{c}$ and ice $I_{h}$ as well as investigate the nature and density of stacking faults. Further experiments are also required to determine if individual frozen droplets contain regions of ice $\mathrm{I}_{\mathrm{h}}$ and ice $I_{c}$ with stacking faults or if individual droplets freeze exclusively to a stacking faulty cubic structure or a hexagonal structure.

The stability of ice $I_{c}$ in water-oil emulsions (i.e. the transformation of ice $I_{c}$ to ice $I_{h}$ in frozen water droplets suspended in an oil matrix).

In this series of experiments the transformation of the ice $I_{c}$ component of the frozen pure water droplets was investigated as a function of time at several temperatures between 228 and $263 \mathrm{~K}$. Emulsified droplets of $d_{\mathrm{vm}} \approx 10 \mu \mathrm{m}$ were cooled at a rate of $10 \mathrm{~K} \mathrm{~min}^{-1}$ to $223 \mathrm{~K}$, and then the temperature was rapidly ramped to the temperature of the isothermal transformation measurement $\left(T_{\text {trans }}\right)$. While the frozen droplets were held at $T_{\text {trans }} \pm 0.2 \mathrm{~K}$, the diffraction pattern between $2 \theta=39.3$ and $44.3^{\circ}$ was monitored. This covers the exclusive ice $\mathrm{I}_{\mathrm{h}}$ reflection at $2 \theta \approx 43.5^{\circ}$ and the reflection common to both ice $I_{\mathrm{c}}$ and ice $\mathrm{I}_{\mathrm{h}}$ at $2 \theta \approx 40^{\circ}$.

The ratio $I_{44} / I_{40}$ determined during the isothermal transformation measurements is plotted in Fig. 4. Prior to starting the isothermal transformation measurements $I_{44} / I_{40}$ was close to 0.4 in all measurements, which indicates that a significant amount of ice $\mathrm{I}_{\mathrm{c}}$ resulted from the freezing process, as expected from the earlier work. The results in Fig. 4 show that at $228 \mathrm{~K}$ ice $I_{c}$ is very stable. When the ice was held at $228 \mathrm{~K}$ the frozen droplets still contained a significant amount at ice $\mathrm{I}_{\mathrm{c}}$ after nearly $12 \mathrm{~h}$, with an intensity ratio $I_{44} / I_{40}=0.56 \pm 0.02$ (recall that $I_{44} / I_{40}$ for stacking faulty ice $\mathrm{I}_{\mathrm{c}}$ is 0 and for pure ice $\mathrm{I}_{\mathrm{h}}$ it is $0.79 \pm 0.03$ ). In fact, even after nearly $5 \mathrm{~h}$ at $238 \mathrm{~K}$ the ice had not fully relaxed to perfect ice $\mathrm{I}_{\mathrm{h}}\left(I_{44} / I_{40}=0.65 \pm 0.03\right)$. In contrast ice $\mathrm{I}_{\mathrm{c}}$ at $263 \mathrm{~K}$ is rapidly converted to ice $\mathrm{I}_{\mathrm{h}}$, and at $243 \mathrm{~K}$, almost all of the ice $\mathrm{I}_{\mathrm{c}}$ is converted to ice $\mathrm{I}_{\mathrm{h}}\left(I_{44} / I_{40}=\right.$ $0.73 \pm 0.05$ ) after $178 \mathrm{~min}$.

To test if X-ray exposure was influencing the ice films during the annealing experiments, we repeated the measurements at $228 \mathrm{~K}$, but this time the shutter to the X-ray source was only opened for enough time to establish the ratio $I_{44} / I_{40}$ once every few hours. The results of this test (not shown) are in agreement within the uncertainty of the measurement with the results presented in Fig. 4 (open triangles) indicating that the expo-

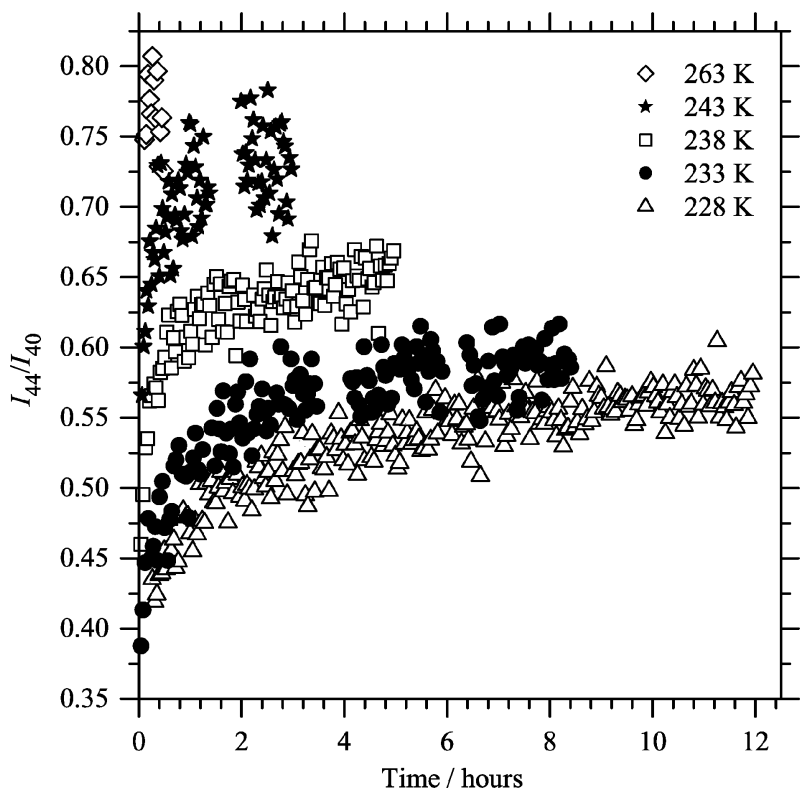

Fig. 4 The intensity ratio $I_{44} / I_{40}$ as a function of time after freezing for pure water droplets $\left(d_{\mathrm{vm}} \approx 10 \mu \mathrm{m}\right)$. A value of $0.79 \pm 0.3$ corresponds to the ratio of ice $\mathrm{I}_{\mathrm{h}}$ with no detectable ice $\mathrm{I}_{\mathrm{c}}$ stacking faults, and a value of zero indicates that no bulk ice $I_{h}$ formed in the droplets, and therefore that the dominant product was ice $\mathrm{I}_{\mathrm{c}}$. Results are shown for several different isothermal transformation temperatures.

sure to X-rays did not significantly affect the ice crystal structure.

Many of the previous measurements of the ice $I_{c}$ to ice $I_{h}$ phase transition have found that ice $I_{c}$ transforms to ice $I_{h}$ rapidly below $\sim 205 \mathrm{~K} .{ }^{23}$ It has been suggested that the ice $\mathrm{I}_{\mathrm{c}}$ to ice $I_{h}$ phase transition is related to surface area - very high surface area ice $I_{c}$ tends to transform more rapidly and at lower temperatures to the stable ice $\mathrm{I}_{\mathrm{h}}{ }^{4}$ In high surface area ice $I_{c}$ samples, surface nucleation is likely to be much more rapid ${ }^{4}$ and also possibly mass transfer from ice $I_{c}$ crystals to ice $I_{h}$ crystals via the gas phase may have dominated in many experiments. ${ }^{2}$ In our experiments the particles are suspended in an oil matrix and hence mass transfer via the gas phase and possibly surface nucleation were blocked, which provides an explanation for the longer lifetimes at higher temperatures observed in our studies.

Our long lifetimes at higher temperatures are consistent with a number of studies of low surface area ice $\mathrm{I}_{\mathrm{c}}$. Using X-ray diffraction Mayer and Hallbrucker ${ }^{4}$ found that ice $I_{c}$ samples, prepared by hyperquenching water droplets, required around 30 min at $240 \mathrm{~K}$ to fully convert to ice $\mathrm{I}_{\mathrm{h}}$. Using a similar technique to produce ice $\mathrm{I}_{\mathrm{c}}$, Kohl et al. ${ }^{3}$ found that the transition was centred around $230 \mathrm{~K}$, while Kuhs et al. ${ }^{15}$ found that a sample of ice $I_{c}$ made from ice $V$ mostly transformed below $205 \mathrm{~K}$, but a significant portion remained in the cubic phase up to between 237 and $245 \mathrm{~K}$. Kuhs et al. suggested this cubic portion of their ice took the form of planar stacking faults within ice $\mathrm{I}_{\mathrm{h}}$. Cubic ice has also been observed at higher temperatures in porous silica. ${ }^{24}$ The stability in these experiments is likely due in part to the confined geometries. 
Also of interest is the nonlinear trend observed in Fig. 4. For example, at $233 \mathrm{~K}$ there is a fast increase in the ratio and then it levels off, indicating that a certain fraction of the cubic ice is very long lived. This is consistent with the measurements of Kuhs et al., ${ }^{15}$ described above in that portions of the ice $\mathrm{I}_{\mathrm{c}}$ appear to be more stable than others. The fact that a similar trend was observed using two very different methods of preparing ice $I_{c}$ suggests that this trend may be an intrinsic property of cubic ice. Possibly related, Johari argued that ice $\mathrm{I}_{\mathrm{c}}$ and $I_{h}$ can coexist over a broad temperature range due to contributions from grain boundaries, interphases and strain energies. $^{25}$ The nonlinear trend observed in Fig. 4 may also be related to slow nucleation kinetics in some frozen droplets. As mentioned we most likely only measured the solid state transition (as the vapour-mediated transformation is blocked). Therefore, the phase transition is likely initiated by nucleation in lattice imperfections, such as line defects and at grain boundaries. ${ }^{26}$ Some frozen droplets may have significantly less imperfections for nucleation of ice $\mathrm{I}_{\mathrm{h}}$, resulting in some frozen droplets with very long lived ice $I_{c}$ regions. Smaller frozen particles may have significantly less imperfections compared to large particles, assuming the occurrence of imperfections is random. In this case, ice $I_{c}$ in the smaller particles will be more stable than in the larger particles (assuming the conversion rate is limited by nucleation at lattice imperfections). Further research is needed on this topic.

\section{Conclusions}

The data presented in this paper are consistent with the emulsified water droplets freezing to a significant proportion of stacking faulty ice $I_{c}$ and that the proportion of stacking faulty ice $I_{c}$ increases as the droplets decrease in size. In fact, droplets of volume median diameter $5.6 \mu \mathrm{m}$ appear to freeze dominantly to ice $I_{c}$ with stacking faults. The size dependence can be rationalised with heat transfer calculations since smaller droplets, with a large surface area to volume ratio, will dissipate heat more rapidly and be less likely to form ice $I_{h}$. These results support previous suggestions that ice $I_{c}$ is the crystalline phase that nucleates when pure water droplets freeze homogeneously at $\sim 235 \mathrm{~K} .^{5-9}$

When water droplets freeze in the atmosphere, our results suggest that ice $I_{c}$ will initially nucleate. However, heat transfer calculations show that when droplets freeze in the atmosphere they will be more likely to freeze to ice $I_{h}$ than droplets in our emulsion experiments. The rate of heat dissipation for a particle suspended in a gas will be roughly a factor of 10 smaller for a similar droplet suspended in oil due to the difference in thermal conductivity of the medium. ${ }^{27}$ According to simple heat transfer calculations, ${ }^{12}$ a $1 \mu \mathrm{m}$ droplet in the atmosphere will have a similar propensity for freezing to ice $I_{c}$ as a $10 \mu \mathrm{m}$ droplet in our experiment. Heymsfield and Miloshevich ${ }^{28}$ found evidence that liquid droplets smaller than $3 \mu \mathrm{m}$ froze homogeneously below $237 \mathrm{~K}$ in orographic wave clouds; we suggest that these droplets may have frozen to a significant amount of ice $I_{c}$.

We observe ice $I_{c}$ up to $243 \mathrm{~K}$, much higher in temperature than observed in many previous studies. ${ }^{23}$ This result adds to the existing literature ${ }^{3,4,15}$ that shows ice $I_{c}$ can persist up to $\sim 240 \mathrm{~K}$. Our results focus on the solid state transformation since we most likely blocked surface nucleation and vapour transport by placing droplets in an oil emulsion in contrast to most previous studies.

The ice $I_{c}$ to ice $I_{h}$ measurements show a complex time and temperature dependence of the phase transition. The transformation proceeds rapidly at first and then slows down and comes to a halt. This could be because a fraction of the frozen droplets have fewer lattice imperfections and hence the ice $I_{c}$ in these particles is more stable. Alternatively, the results may in part be due to ice $I_{c}$ stacking sequences that persist at temperatures above $205 \mathrm{~K}^{15}$

The results from this study, combined with results from other studies, help explain why ice $\mathrm{I}_{\mathrm{c}}$ is observed in some bulk samples and not others: Ice $\mathrm{I}_{\mathrm{c}}$ must always be held below $\sim 240 \mathrm{~K}$ regardless of the surface area of the sample or else it will be rapidly converted to ice $I_{h}$ through a solid state tranformation. (One exception is ice $I_{c}$ formed in nanoporous material, which can exist to higher temperatures, possibly due to the confined geometries. ${ }^{24}$ ) Also, during the crystallization process, the rate of heat dissipation from the sample needs to be greater than the rate of heat production by crystallization or else the sample will heat up and the ice $I_{c}$ may be converted to ice $\mathrm{I}_{\mathrm{h}}$. In addition, if the ice has a high surface area and mass transfer via the vapour is not blocked, it must be prepared below $\sim 200 \mathrm{~K}$, or else surface nucleation and mass transfer will occur and any ice $\mathrm{I}_{\mathrm{c}}$ that forms will be converted rapidly to ice $I_{h}$, by vapour-mediated transformation. This overall discussion is consistent with our measurements as well as most measurements of ice $I_{c}$ reported in the literature. ${ }^{3,4,13,23}$

\section{Acknowledgements}

We thank A. Lam and B. Patrick for their assistance with the $\mathrm{X}$-ray diffraction measurements and the interpretation of the diffraction patterns and R. Signorell for helpful comments on the manuscript. This work was funded by the Canadian Foundation for Climate and Atmospheric Sciences, CFCAS, the Natural Science and Engineering Research Council of Canada, NSERC, the Canada Foundation for Innovation, CFI, and the Canada Research Chairs Program.

\section{References}

1 B. J. Murray, D. A. Knopf and A. K. Bertram, Nature, 2005, 434, 202-205.

2 D. M. Murphy, Geophys. Res. Lett., 2003, 30.

3 I. Kohl, E. Mayer and A. Hallbrucker, Phys. Chem. Chem. Phys., 2000, 2, 1579-1586.

4 E. Mayer and A. Hallbrucker, Nature, 1987, 325, 601-602.

5 J. F. Huang and L. S. Bartell, J. Phys. Chem., 1995, 99, 3924-3931.

6 Y. Furukawa, J. Met. Soc. Japan, 1982, 60, 535-547.

7 T. Takahashi and T. Kobayashi, J. Cryst. Growth, 1983, 64, 593-603.

8 H. Kiefte, M. J. Clouter and E. Whalley, J. Chem. Phys., 1984, 81, 1419-1420.

9 L. S. Bartell and Y. G. Chushak, in Water in Confined Geometries, ed. V. Buch and J. P. Devlin, Springer-Verlag, Berlin, 2003, pp. 399-424.

10 W. Kraus and G. Nolze, J. Appl. Crystallogr., 1996, 29, 301-303.

11 A. Goto, T. Hondoh and S. Mae, J. Chem. Phys., 1990, 93, $1412-1417$. 
12 H. R. Pruppacher and J. D. Klett, Microphysics of Clouds and Precipitation, Kluwer, Dordrecht, 1997.

13 L. G. Dowell and A. P. Rinfret, Nature, 1960, 188, 1144-1148.

14 W. F. Kuhs, D. V. Bliss and J. L. Finney, J. Phys. Colloq., 1987, 48, 631-636.

15 W. F. Kuhs, G. Genov, D. K. Staykova and T. Hansen, Phys. Chem. Chem. Phys., 2004, 6, 4917-4920.

16 G. P. Arnold, E. D. Finch, S. Rabideau, W. and R. G. Wenzel, J. Chem. Phys., 1968, 49, 4365-4369.

17 A. I. Ustinov, in Defect and Microstructure Analysis by Diffraction, ed. R. Snyder, J. Fiala and H. J. Bunge, Oxford University Press, Oxford, 1999, pp. 264-317.

18 Z. Weiss and P. Capková, in Defect and Microstructure Analysis by Diffraction, ed. R. Snyder, J. Fiala and H. J. Bunge, Oxford University Press, Oxford, 1999, pp. 318-329.
19 P. C. Reist, Aerosol Science and Technology, McGraw-Hill, New York, 1993.

20 G. P. Johari, J. Chem. Phys., 2005, 122.

21 M. Matsumoto, S. Saito and I. Ohmine, Nature, 2002, 416, 409-413.

22 T. Takahashi, J. Cryst. Growth, 1982, 59, 441-449.

23 P. Hobbs, Ice Physics, Oxford University Press, London, 1974.

24 D. C. Steytler, J. C. Dore and C. J. Wright, J. Phys. Chem., 1983, 87, 2458-2459.

25 G. P. Johari, Philos. Mag. B, 1998, 78, 375-383.

26 Y. V. Mnyukh and N. A. Panfilova, J. Phys. Chem. Solids, 1973, 34, 159-170.

27 R. C. Weast, Handbook of Physics and Chemistry, CRC Press, Boca Raton, 70th edn, 1990.

28 A. J. Heymsfield and L. M. Miloshevich, J. Atmos. Sci., 1993, 50, 2335-2353. 